Mathias Junginger | Christian Kübel | Felix H. Schacher Axel H. E. Müller | Andreas Taubert

\title{
Crystal structure and chemical composition of biomimetic calcium phosphate nanofibers
}

Suggested citation referring to the original publication:

RSC Advances 3 (2013), pp. 11301-11308

DOI http://dx.doi.org/10.1039/c3ra23348k

Postprint archived at the Institutional Repository of the Potsdam University in:

Postprints der Universität Potsdam

Mathematisch-Naturwissenschaftliche Reihe ; 244

ISSN 1866-8372

http://nbn-resolving.de/urn:nbn:de:kobv:517-opus4-95176 

Cite this: RSC Advances, 2013, 3, 11301

Received 15th December 2012, Accepted 1st May 2013

DOI: $10.1039 / \mathrm{c} 3 \mathrm{ra} 23348 \mathrm{k}$

www.rsc.org/advances

\section{Crystal structure and chemical composition of biomimetic calcium phosphate nanofibers}

\author{
Mathias Junginger, ${ }^{a}$ Christian Kübel, ${ }^{\text {bc }}$ Felix H. Schacher, ${ }^{d}$ Axel. H. E. Müllerte \\ and Andreas Taubert*a
}

\begin{abstract}
Calcium phosphate nanofibers with a diameter of only a few nanometers and a cotton-ball-like aggregate morphology have been reported several times in the literature. Although fiber formation seems reproducible in a variety of conditions, the crystal structure and chemical composition of the fibers have been elusive. Using scanning transmission electron microscopy, low dose electron (nano)diffraction, energy-dispersive X-ray spectroscopy, and energy-filtered transmission electron microscopy, we have assigned crystal structures and chemical compositions to the fibers. Moreover, we demonstrate that the mineralization process yields true polymer/calcium phosphate hybrid materials where the block copolymer template is closely associated with the calcium phosphate.
\end{abstract}

\section{Introduction}

Calcium phosphate is one of the most important biominerals and has attracted much attention from the biomaterials community. ${ }^{1-5}$ Bioinspired or biomimetic growth of calcium phosphate from aqueous solution at roughly physiological conditions has been studied for the synthesis of new biomaterials with properties that are much closer to those of natural materials than many of the more traditional biomaterials such as ceramics or metals. Polymer-controlled mineralization has also been used as model for understanding and quantifying calcium phosphate biomineralization. ${ }^{6-10}$

Most studies focus on calcium phosphate mineralization from bulk aqueous solution. ${ }^{9}$ In contrast, there are only few studies on the effects of interfaces on calcium phosphate nucleation and growth, although the recent past has seen an increasing number of articles on the topic. ${ }^{11-19}$ Likewise, the effects of (poly)cations on calcium phosphate and calcium carbonate mineralization have only recently begun to be addressed. ${ }^{13,14,19-26}$ According to these studies, there are indeed significant differences between (i) bulk and surface mineralization and between (ii) polyanion and polycation-

${ }^{a}$ Institute of Chemistry, University of Potsdam, Karl-Liebknecht-Str. 24-25, D-14476 Potsdam, Germany. E-mail: ataubert@uni-potsdam.de

${ }^{b}$ Karlsruhe Institute of Technology, Institute of Nanotechnology, Hermann-vonHelmholtz-Platz 1, D-76344 Eggenstein-Leopoldshafen, Germany

${ }^{c}$ Karlsruhe Institute of Technology, Karlsruhe Nano Micro Facility, Hermann-vonHelmholtz-Platz 1, D-76344 Eggenstein-Leopoldshafen, Germany

${ }^{d}$ Institut für Organische Chemie und Makromolekulare Chemie, Friedrich-SchillerUniversität Jena, Lessingstr. 8, D-07743 Jena, Germany

${ }^{e}$ Makromolekulare Chemie II, University of Bayreuth, Naturwissenschaften II (NW II), D-95440 Bayreuth, Germany

† Current address: Institute of Organic Chemistry, Johannes Gutenberg

University Mainz, Duesbergweg 10-14, D-55099 Mainz, Germany. controlled calcium phosphate mineralization. Among others, poly(cation) monolayers at the air-water interface template the formation of intriguing fibers with diameters of only a few nanometers. ${ }^{13,14}$ As the fibers are many micrometers long and form extended structures, they could be interesting for the construction of hybrid biomaterials or as fillers for mechanical stabilization of composite biomaterials. Interestingly, the fibers resemble examples that have been made via precipitation from bulk solution using negatively charged polymer additives. ${ }^{27-30}$

Peytcheva et $a .^{28}$ used a triple-jet setup where $\mathrm{CaCl}_{2}$, $\mathrm{Na}_{2} \mathrm{HPO}_{4}$, and sodium poly(D-L-aspartate) solutions were continuously added to a sodium poly(D-L-aspartate) solution. Nanofibers were obtained between $\mathrm{pH} 6.9$ and 8.1. Antonietti et $a .^{27}$ used an approach where the double-hydrophilic block copolymer (DHBC) poly(ethylene oxide)-block-alkylated poly (methacrylic acid) was dissolved in a $0.68 \mathrm{M} \mathrm{CaCl}_{2}$ solution. Subsequently, stoichiometric amounts of $\mathrm{NaH}_{2} \mathrm{PO}_{4} / \mathrm{Na}_{2} \mathrm{HPO}_{4}$ solutions with a preset $\mathrm{pH}$ were added. At $\mathrm{pH}$ values between 3.5 and 5, the authors observed the formation of what they called neuron-like calcium phosphate/polymer mesostructures. Tjandra et $a l^{29,30}$ used the DHBC poly(ethylene oxide)block-poly(methacrylic acid) similar to Antonietti et $a .^{27}$ and obtained similar mineral morphologies. The most obvious similarity of these data is the fact that the fibers were in all cases obtained at low to medium pH (3 to $c a$. 8) where usually thermodynamically less stable calcium phosphates such as brushite are obtained. ${ }^{9,31}$ In spite of this, one study $^{28}$ found that the precipitates contained a significant fraction of hydroxyapatite even when the $\mathrm{pH}$ of the reaction mixture was lower than 8 . This is surprising because HAP typically only precipitates above a $\mathrm{pH}$ of $c a .8$ to $8.5 .^{9,31}$ 
This raises two questions: (i) are the assignments of the crystal structures given in the literature correct and, if yes, (ii) how does HAP form at such low $\mathrm{pH}$ values? The implication is that, if the precipitates obtained at these low $\mathrm{pH}$ values indeed consist of HAP, the models of calcium phosphate mineralization, in particular the concepts of (crystal) phase selection, must be modified to account for the apparent discrepancy between long established (and well-confirmed) views ${ }^{31}$ and the observation that even at low $\mathrm{pH}$ (possibly as low as $\mathrm{pH} 3.5)^{27}$ HAP can form, provided that the right template is available during mineralization. To address this question, however, more quantitative data on the crystal structures and the chemical composition of the nanofibers are needed.

The main reason for the uncertainty on the structural and chemical details of these fibers is their very low stability, which prevents the investigation with techniques such as selected area electron diffraction (SAED), high resolution TEM (HRTEM), or other methods commonly used for nanomaterials analysis. This has limited fiber analysis to a fairly qualitative morphology description and a number of unconfirmed assumptions. Only Peytcheva et $a .^{28}$ have proposed a structure (HAP with the $c$-axis along the fiber axis), but the proposal has so far not been backed up with experimental or theoretical data. A further complication arises from the low amounts of material that are typically recovered from an experiment. This is a challenge for both the materials analysis and the translation into technologically relevant materials.

The current article therefore concentrates on the structural and chemical analysis of nanofibers synthesized via interfacial mineralization of calcium phosphate beneath monolayers of poly(n-butylmethacrylate)-block-poly[2-(dimethylamino)ethyl methacrylate] block copolymers at the air-water interface. ${ }^{14}$ By combination of high angle annular dark field scanning transmission electron microscopy (HAADF-STEM), energyfiltered TEM (EFTEM), nano-electron diffraction (NED), and energy-dispersive X-ray spectroscopy (EDXS) new information on the crystal structure and the chemical composition of the nanofibers is now available.

\section{Experimental}

\section{Materials}

Monosodium phosphate, calcium chloride trihydrate (Roth), and chloroform (spectroscopic grade, Merck) were used as received. All glassware was treated with a $\mathrm{H}_{2} \mathrm{O}_{2} / \mathrm{H}_{2} \mathrm{SO}_{4}(1: 2)$ mixture and rinsed with doubly distilled water before use. Water with a resistivity of $18.2 \mathrm{M} \Omega \mathrm{cm}$ for Langmuir-Blodgett experiments was obtained from a Millipore MilliQ® setup. The synthesis of the block copolymer used in this study, poly (n-butylmethacrylate)-block-poly[2-(dimethylamino)ethyl methacrylate] PnBMA P5 $^{-}$PDMAEMA $_{147}\left(33.700 \mathrm{~g} \mathrm{~mol}^{-1}\right.$, PDI 1.10, the subscripts denote the degrees of polymerization for the corresponding segment) has been described before. ${ }^{14}$

\section{Mineralization}

Mineralization experiments were conducted on a Nima Technology (UK) medium deposition trough with two moveable barriers. The trough was placed on an anti-vibration table in a dust-free room. All experiments were done at room temperature. For mineralization, the block copolymer was spread from chloroform $\left(1.1 \mathrm{mg} \mathrm{mL}^{-1}\right)$ on $180 \mathrm{~mL}$ of a $20 \mathrm{mM}$ $\mathrm{NaH}_{2} \mathrm{PO}_{4}$ aqueous subphase with pH $5 \pm 0.1$. After chloroform evaporation the polymer film was compressed to $30 \mathrm{mN} \mathrm{m}^{-1}$ and $1.8 \mathrm{~mL}$ of an aqueous $2 \mathrm{M} \mathrm{CaCl}_{2}$ solution were injected into the subphase via a Hamilton syringe. The calcium and phosphate concentrations were chosen to enable comparison with our previous studies and to avoid a "pre-programming" of HAP formation into the mineralization reaction by deliberately using mixtures with a $\mathrm{Ca} / \mathrm{P}$ ratio of 1.67 . The subphase was slowly and continuously stirred by placing a small stirring rod in the dipping well of the trough. Samples were collected after 4 to $8 \mathrm{~h}$ of mineralization. The mineralization reaction has been reproduced at least three times for both subphase conditions ( 2 and $20 \mathrm{mM}$ ). The data between the samples are consistent and the images shown are a selection from data on different samples.

\section{Transmission electron microscopy}

Transmission electron microscopy was done on a CM100 (Philips, Eindhoven, NL) operated at $80 \mathrm{kV}$ and on an imagecorrected FEI Titan 80-300 (FEI Company, Hillsborough, USA) operated at $300 \mathrm{kV}$ equipped with a US1000 (Gatan) slowscan CCD camera, a Tridium image filter (Gatan), a high-angle annular dark-field (HAADF) detector for scanning transmission electron microscopy (STEM), and a S-UTW EDX detector (EDAX). TEM samples were prepared by film transfer ${ }^{17}$ onto holey carbon films uniformly coated with an additional $2 \mathrm{~nm}$ of carbon (Quantifoil $\AA$ Holey Carbon Films + 2 nm C, Au 200 mesh) and drying in air.

\section{Results}

To simplify the fiber synthesis we have, in contrast to our previous studies, ${ }^{13,14}$ not buffered the reaction mixture, but used the same block copolymer and the same salt concentrations ( $2 \mathrm{mM}$ ) as in the previous work. Bright field transmission electron microscopy (BF-TEM) shows that this does not affect the outcome of the mineralization as in all cases fibers with diameters of a few nanometers form. Extended electron microscopy tests, however, showed that these fibers are not suitable for a detailed structural investigation: in accordance with our previous results, ${ }^{13,14}$ the fibers obtained from $2 \mathrm{mM}$ solutions are sensitive to electron irradiation to a point that any electron microscopic analysis other than bright field imaging at low magnifications becomes impossible. That is, no crystallographic information or data on the chemical composition of these fibers can be obtained, even under low dose conditions.

For the current study, we have thus modified the mineralization protocol (see experimental part for details) and increased both the calcium and phosphate concentrations to 

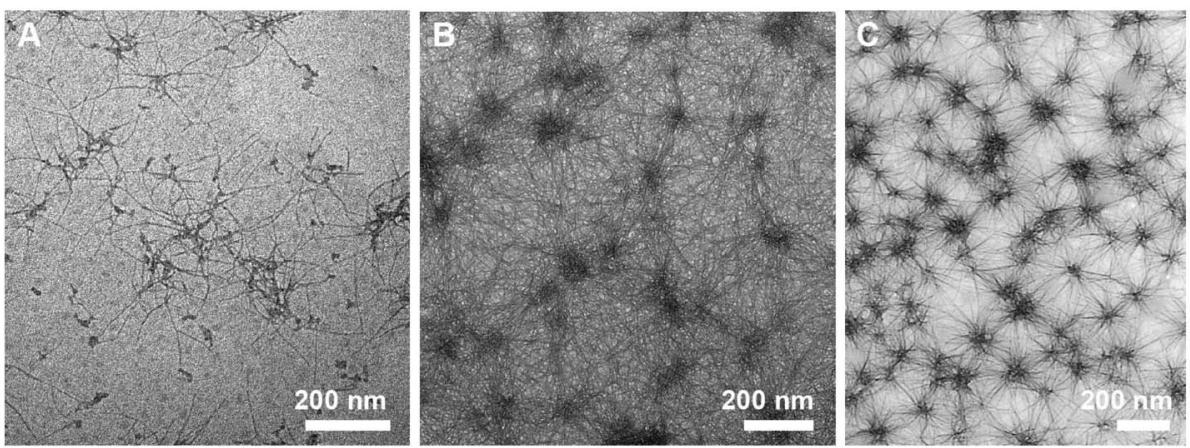

Fig. 1 Bright field transmission electron microscopy (BF-TEM) images of (A) sample precipitated from $2 \mathrm{mM}$ solution, (B) sample obtained from $20 \mathrm{mM}$ solution (zeroloss filtered image), (C) nested structures obtained by Antonietti et al. ${ }^{27}$ Note that the mineralization conditions in our previous studies ${ }^{13,14}$ are slightly different than in the current work; this leads to the somewhat different fiber morphology observed in panel (A) compared to data shown in our earlier publications. ${ }^{13,14}$ Image shown in panel (C) was provided by Prof. Helmut Cölfen, University of Konstanz.

$20 \mathrm{mM}$. The resulting fibers are morphologically very similar to those obtained previously (diameters below ca. $5 \mathrm{~nm}$ and lengths in the micrometer range) and to fibers reported in the literature, ${ }^{27,28}$ but BF-TEM shows that mineralization from 20 $\mathrm{mM}$ solutions leads to more electron beam-resistant fibers and to a much higher number of fibers than mineralization from 2 $\mathrm{mM}$ solutions ${ }^{13,14}$ (Fig. 1). We currently assign the higher stability towards the electron beam to a higher degree of mineralization.

Mineralization from $20 \mathrm{mM}$ solutions leads to fibers covering large areas of the TEM grid, including tightly interconnected dense areas that have been termed "nested structures" by Antonietti et al. (Fig. 1C). ${ }^{27}$ These structures have also been observed in a few other studies. ${ }^{28-30}$ We therefore infer that the fibers formed at $20 \mathrm{mM}$ at the PnBMA $_{75}$-PDMAEMA 147 block copolymer monolayers are chemically and crystallographically closely related to those reported previously. ${ }^{13,14,27-30}$

Fig. 2 shows high angle annular dark field scanning transmission electron microscopy (HAADF-STEM) and energy dispersive X-ray spectroscopy (EDXS) data obtained from the fibers and nested structures. Because the contrast in HAADFSTEM approximately scales with $Z^{1.7}$ to $Z^{2}$ it is an ideal technique for imaging elements with high atomic number $(Z)$ in a low $Z$ background. ${ }^{32,33}$ Regions containing heavy elements appear bright in HAADF-STEM and, for example, already the contrast between relatively light elements like $\operatorname{Si}(Z=12)$, Co $(Z$ $=27), \mathrm{Zn}(Z=30)$ and a polymeric matrix (which essentially is carbon, $Z=6$ ) is sufficiently large to even image regions with only a few heavy elements in a small feature of a few $\mathrm{nm}$ in diameter. $^{34,35}$ In the current case, the high contrast between the fibers and the background is therefore assigned to the deposition of calcium phosphate (assuming the formation of stoichiometric HAP Ca $\left(\mathrm{PO}_{4}\right)_{3} \mathrm{OH}$, the average value of $Z$ would be 13) on a carbon/oxygen/nitrogen background (the block copolymer monolayer, average $Z \approx 6.15$ ). HAADF-STEM therefore supports the earlier claims of calcium phosphate fiber formation, ${ }^{13,14,27-30}$ although HAADF-STEM does not allow the determination of a crystal phase.
Consistent with HAADF-STEM, all EDX spectra show calcium, phosphorus, oxygen, and carbon signals. The calcium, phosphorus, and oxygen signals can be assigned to calcium phosphate with a small contribution to the oxygen signal from the polymer template and the $2 \mathrm{~nm}$-support film on the TEM grid. The carbon signals can be assigned to the carbon support films on the TEM grid and the polymeric template. Quantitative EDX spectroscopy on multiple areas of the grids finds a $\mathrm{Ca} / \mathrm{P}$ ratio of $1.17 \pm 0.09$. This ratio is close to

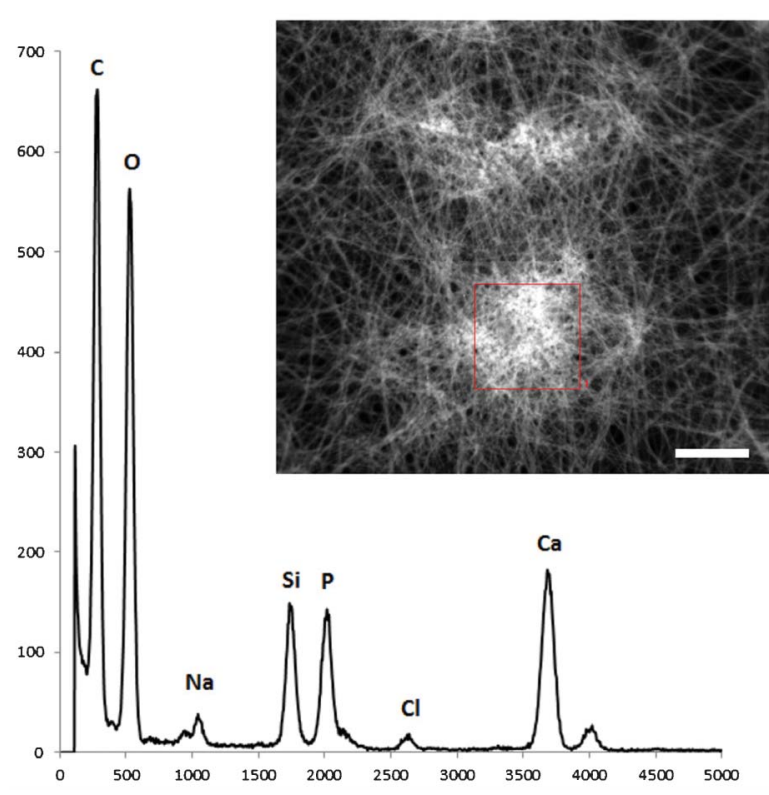

Fig. 2 Integrated HAADF-STEM-EDX measurement of fibrillar structures. Main panel: EDX spectrum (average of 7 different areas). The Si signal varies from sample to sample and likely stems from a contamination late in the experiments, because earlier studies did not show Si contamination. ${ }^{14}$ For example, small amounts of silicone grease used during any time of the sample preparation or transport tends to deposit on the support and decomposes during TEM measurements accumulating in the area used for the measurement. However, this only adds carbon, oxygen and silicon to the EDX measurement, but leaves the $\mathrm{Ca} / \mathrm{P}$ ratio unchanged. Inset: HAADF-STEM image of one region used for EDX spectrum acquisition (red frame). Scale bar is $100 \mathrm{~nm}$. 
brushite (DCPD, 1.0) or monetite (DCPA, 1.0). Monetite forms at higher temperatures ${ }^{9,31}$ but could also form in a special microenvironment at room temperature. However, the electron diffraction patterns (see below) could not be matched with monetite, so the presence of monetite is less likely. Octacalcium phosphate (OCP, 1.33) and hydroxyapatite (HAP, 1.67) have higher $\mathrm{Ca} / \mathrm{P}$ ratios, ${ }^{2,9,31}$ but there are numerous examples where these phases exist in substoichiometric forms. ${ }^{9,31}$ EDXS therefore indicates the formation of DCPD with OCP or HAP as a second component.

Besides the expected $\mathrm{Ca}, \mathrm{P}, \mathrm{O}$, and $\mathrm{C}$ signals, also signals of $\mathrm{Na}$ and $\mathrm{Cl}$ were consistently observed. Based on the EDX quantification, the combined $\mathrm{Na}$ and $\mathrm{Cl}$ fraction in the material is about $3 \%$. The signals could originate from $\mathrm{NaCl}$, which may deposit on the polymer monolayers used as templates for the mineralization reaction. Both $\mathrm{Na}$ and $\mathrm{Cl}$ are present in the original mineralization medium used for calcium phosphate formation (from the precursor salts $\mathrm{CaCl}_{2}$ and $\mathrm{NaH}_{2} \mathrm{PO}_{4}$ ) and assuming the formation of rock salt is therefore straightforward. We have indeed occasionally observed $\mathrm{NaCl}$ nanocrystals in the TEM; their crystal structure has been confirmed by electron diffraction.

Alternatively, the formation of clorapatite (ClAP) and sodium-substituted calcium phosphates is also possible. Both the formation of ClAP, a close relative of HAP, and substitution of the apatite lattice with sodium are known in biomineralization and in vitro mineralization. ${ }^{9,31}$ As we only rarely observe the formation of $\mathrm{NaCl}$, we currently speculate that $\mathrm{Na}$ and $\mathrm{Cl}$ could be incorporated in the apatite phases. Electron diffraction is however not accurate enough to distinguish between these different types of substitution. Because the mineralization experiments do not provide enough material for an in-depth X-ray analysis of the precipitates, we cannot comment on the substitution any further.

To further confirm the assignment of the HAADF-STEM data, we have recorded energy filtered transmission electron microscopy (EFTEM) data from the fibers. EFTEM enables the rapid acquisition of elemental maps in TEM thin sections. It is particularly efficient for light elements and, unlike HAADFSTEM, enables to separately determine the distribution of multiple elements within one and the same sample area. ${ }^{33}$ Fig. 3 shows a representative zero-loss filtered (ZLF) image along with the corresponding $\mathrm{C}, \mathrm{Ca}$, and $\mathrm{P}$ distributions obtained using three-window EFTEM mapping. While the ZLF image is similar to the images shown above, the elemental maps exhibit a fairly poor signal-to-noise ratio. The reason for this is again the radiation sensitivity of the material together with the small inelastic scattering cross-section. The EFTEM maps were acquired limiting the dose, just so that no significant difference in sample morphology was visible in the ZLF images before and after acquiring the EFTEM maps. Despite the low signal-to-noise ratio, the $\mathrm{Ca}$ and $\mathrm{P}$ distributions closely resemble each other and show the association of calcium and phosphate in the fibers. The $\mathrm{C}$ map is less clear and the features appear blurry. This indicates that the fibers are embedded in a polymer matrix or located at the polymer film that has been transferred to the TEM grid.

EFTEM and HAADF-STEM-EDX show that the fibers are real organic/inorganic hybrid materials. One of the obvious key questions, however, is the crystal structure (if any) of the deposited calcium phosphate. Fig. 4 shows a representative nano-area electron diffraction (NED) pattern obtained from larger regions of the fibrous material. All patterns exhibit weak diffraction rings, some of them fairly sharp. Such patterns can be assigned to well-crystallized but polycrystalline samples. NED therefore suggests that the fiber-like features are indeed crystalline. Moreover, the fact that some of the rings are rather sharp indicates that the crystallinity is quite high.

The NED patterns exhibit three intense rings corresponding to $d$-spacings of 2.98, 2.78 and $2.10 \AA$ and three weak, but fairly sharp reflections at $2.43,1.49$ and $1.33 \AA$. In addition, there are broad poorly defined diffraction rings around 3.5-3.1 and 2.15-1.75 A. Comparison of the $d$-spacings of DCPD, DCPA, HAP, ClAP (chlorapatite), carbonated apatite (CAP), and OCP with the observed $d$-spacings of the fibers suggests that the fibers are (i) strongly textured and (ii) that there is more than one calcium phosphate phase present in the fibers.

The observation of broad reflections is consistent with the fact that the fibers have small nanoscale sizes in two directions, whereas the sharper reflections presumably correspond to the crystal packing along the fiber axis. With the highly anisotropic structure and a number of different possible compositions, it is not possible to uniquely assign all observed reflections. Nevertheless, in simulated powder patterns with strong preferential orientation, we could only produce the high intensity peak at $2.98 \AA$ for DCPD, whereas the high intensity peak at $2.78 \AA$ can only be reproduced for HAP or OCP. This suggests that the fibers consist of significant amounts of DCPD with HAP and/or OCP also present. Finally, the broad background could also indicate the presence of amorphous calcium phosphate.

Clearly, high resolution TEM (HRTEM) experiments would add further information, but in spite of numerous attempts to obtain HRTEM data, the fibers have proved to be too unstable for HRTEM. This is mostly due to the fact that the NED experiments were performed at relatively low magnifications, while for the HRTEM experiments higher magnifications were needed; at these conditions, the total electron dose was too high for damage-free imaging.

\section{Discussion}

There are several examples of biomimetically grown nanofibers that were reported to be calcium phosphate. ${ }^{13,14,27-30}$ For reasons of material stability, details of the crystal structure and chemical composition have so far not been accessible, although based on XRD data obtained on dried samples, it has been suggested that the fibers consist of HAP. ${ }^{27,28}$ The current study addresses these points and proposes the most detailed structure assignment of said fibers to date. Before 

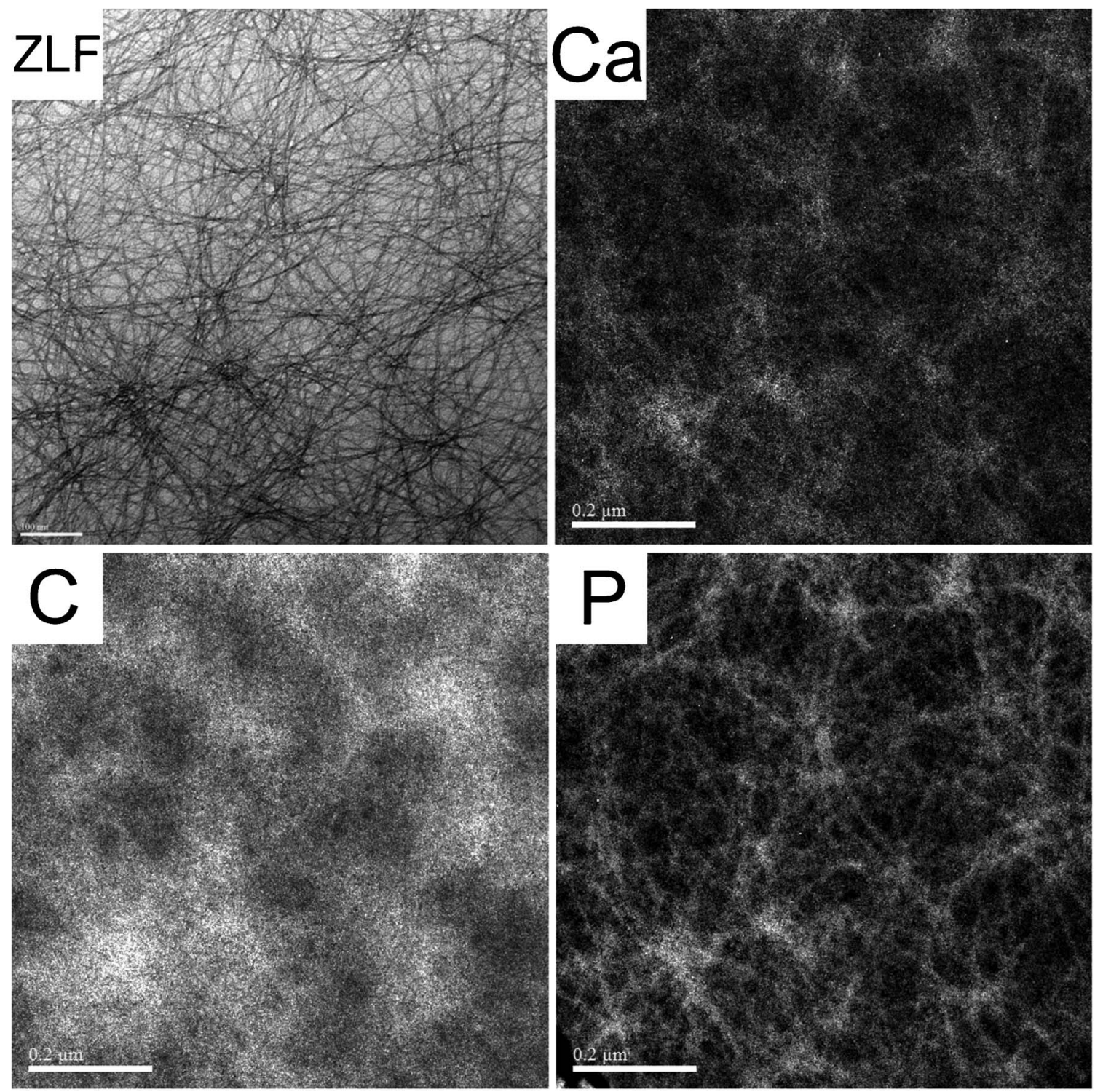

Fig. 3 EFTEM images of the nanofibers. ZLF shows the zero-loss filtered image of the target area. Ca, P, and C show the elemental maps of calcium, phosphorus, and carbon. Brighter areas indicate the presence of larger amounts of the respective element than the dark areas.

delving into a detailed discussion, however, it must clearly be stated that sample isolation and drying prior to TEM imaging may induce structural changes in the samples. The details of sample preparation (such as concentration gradients or $\mathrm{pH}$ changes during drying before TEM experiments) on sample structure will need to be addressed in the future, possibly via cryo-TEM experiments. In spite of this, the fact that the fibers have been reported numerous times under different preparation conditions, ${ }^{13,14,27-30}$ shows that they are indeed real features.

While the fibers are thus recognized as real features throughout the literature, ${ }^{13,14,27-30}$ the situation is more complex in terms of the calcium phosphate phase assignment. This is due to the fact that (i) the calcium phosphate phases are not easy to identify and (ii) again the drying process during sample preparation may induce artifacts. In spite of this, the current data provide the most accurate structural assignment of the calcium phosphate fibers to date; details may have to be revised in the future. For example our assumption of only orthophosphates being present in the samples may not necessarily be entirely correct. Possibly, some fraction of the fibers is made up of amorphous calcium phosphate, which could be the source of the diffuse background scattering in the electron diffraction patterns. However, the intensity and sharpness of the electron diffraction rings in the NED patterns clearly suggests that the majority of the samples is crystalline. Hence, the assumption of orthophosphates as the major calcium phosphate components is feasible.

HAADF-STEM (Fig. 2), EDXS (Fig. 2), and EFTEM (Fig. 3) prove that the fibers contain a high fraction of calcium and 

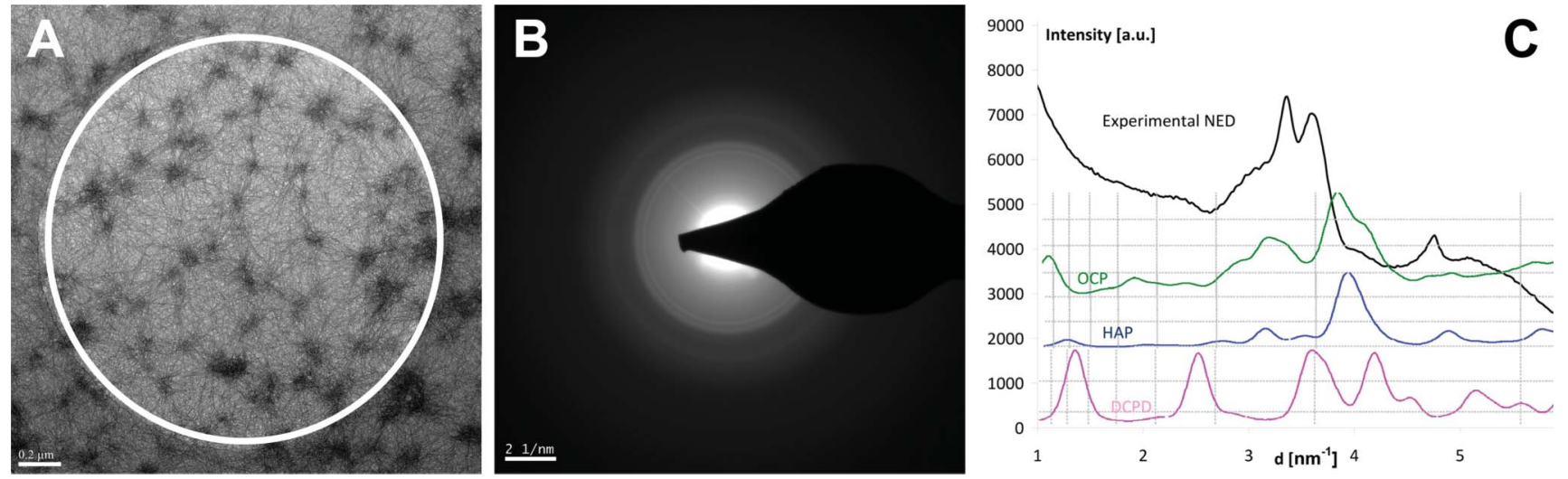

Fig. 4 BF-TEM image (A) and nano-area electron diffraction (NED) pattern (B) of the nanofibers. The white circle in panel (A) indicates the area selected for the

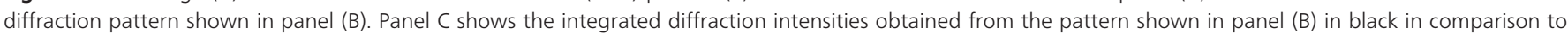
ASTM based simulated X-ray diffraction patterns with diffraction broadening to reflect the small diameter of the fibers.

phosphorus (i.e. phosphate) which are closely associated. These data confirm the formation of calcium phosphate nanofibers. Quantitative EDXS finds a Ca/P $1.17 \pm 0.09$, which is between DCPD and OCP, suggesting that one of the components is DCPD, because the $\mathrm{Ca} / \mathrm{P}$ ratios of other calcium phosphates such as HAP are even higher., ${ }^{9,31}$ Electron diffraction data (Fig. 4) are complex, but confirm the presence of crystalline calcium phosphates. The diffraction patterns suggest that up to three (DCPD, OCP, HAP) different calcium phosphate phases are present in the nanofibers. The presence of HAP has indeed been postulated based on wide angle X-ray scattering data. $^{28}$ Ref. 28 also reported on two strong reflections indicative of brushite, but the authors assigned the brushite phase to nanoplatelets that existed in their samples besides the fibers. In contrast, we also observe reflections indicative of brushite without platelets. This suggests that the brushite crystal phase is an integral part of the observed fibers.

Brushite forms at the lowest $\mathrm{pH}$ values of the three phases, followed by OCP and finally HAP, which typically forms at $\mathrm{pH}$ values of at least $c a .8$ (although HAP can also form at lower $\mathrm{pH}$ values, for example in bone). The presence of brushite thus suggests that OCP likely is also present, because then the actual pH of precipitation would be in between those favoring DCPD and OCP precipitation, with minor fractions of HAP. This would also account for the $\mathrm{Ca} / \mathrm{P}$ ratio of 1.17 , which is between that of DCPD and OCP.

The chemical nature of the polymeric additives must be considered when trying to understand fiber formation on a (supra)molecular level. While older examples of calcium phosphate nanofibers have been synthesized using acidic carboxylate (co)polymers as additives, ${ }^{27-30}$ more recent examples have been made using block copolymers containing basic poly(amine) segments. ${ }^{13,14}$ The interesting point is therefore whether the hypothesis by Peytcheva et $a l^{28}$ (adsorption of anionic polymers on HAP crystal faces where predominantly $\mathrm{Ca}^{2+}$ is exposed; this adsorption leads to a fiber or needle-like morphology where the long axis is the HAP $c$-axis) is the complete explanation for the formation of crystalline calcium phosphate nanofibers.

The explanation is certainly consistent with chemical knowledge in that anionic polymeric additives will undergo electrostatic interaction with cationic surface sites. However, fibers are also observed when polycations are used as crystallization additives. ${ }^{13,14}$ Adsorption to cationic HAP surface sites seems unrealistic and calls for an extended hypothesis.

Instead of polymer adsorption on existing crystal surfaces, ${ }^{28}$ the polymeric additive (positively or negatively charged) can also interact with calcium or phosphate ions prior to nucleation and mineral formation. Previous data on polycation-mediated calcium phosphate mineralization ${ }^{13,14,21}$ indeed indicate that polymer-ion association may be the key step in the mineralization process (this is also supported by new data on calcium carbonate ${ }^{36}$ and $\mathrm{CuO}_{x}$ mineralization ${ }^{37}$ ). At $\mathrm{pH} 5$, where the reactions for the current studies were conducted, the PDMAEMA block is fully protonated (that is, positively charged and fully stretched $)^{13}$ and therefore likely associates with the phosphate ions present in solution. As a result, the fibrous architecture of the final precipitate, as shown in Fig. 1 may already be "pre-programmed" into the polymer-phosphate complexes before any nucleation occurs. The addition of calcium then leads to the fixation of these structures via the formation of fiberlike calcium phosphate deposits along the pre-organized, phosphate-enriched block copolymer chains. Such a model is also applicable to the older examples of calcium phosphate fibers. ${ }^{27-30}$ In these cases, the polymer (an anionic polycarboxylate) coordinates calcium ions along the stretched polymer chain. Addition of phosphate ions will again lead to calcium phosphate fibers along the stretched polymer-calcium ion complexes.

A final point to address is the crystal phases observed in the current study. As stated above, the bulk $\mathrm{pH}$ of the reaction mixture used for fiber synthesis was 5 . At this $\mathrm{pH}$ the formation of OCP or HAP cannot readily be expected ${ }^{31}$ yet we observe evidence for OCP and HAP (Fig. 2 and 4). Indeed, 
we have previously observed that HAP can also form at $\mathrm{pH}$ values where it can normally not be expected (even though there are cases where HAP forms at $\mathrm{pH}$ values lower than 8 , for example in bone). ${ }^{22,38}$ The most prominent example is the transformation of DCPD into HAP in the presence of basic poly(ethylene imine) (PEI). ${ }^{38}$ There, the DCPD-HAP phase transition is much faster than in the presence of acidic additives. This has been assigned to the ability of PEI to take up protons and possibly to locally (on a microscopic length scale) shift the $\mathrm{pH}$ to higher values, which would favor HAP formation. Previous ${ }^{19}$ and current data support these observations because also here, we observe evidence for HAP, which should not form at $\mathrm{pH} 5{ }^{31}$ Although the hypothesis seems feasible, there is a need to locally probe the $\mathrm{pH}$ values close to the block copolymer backbone and further away from the polymer to quantitatively establish the presence and magnitude of the $\mathrm{pH}$ differences during calcium phosphate mineralization.

Overall, the current data show that calcium phosphate nanofibers are crystalline fibers consisting of a set of phases of different stability (DCPD, OCP, HAP), which are closely associated with the block copolymer template and are highly anisotropic in shape. Moreover, the data suggest that the fiber formation is possibly not due to polymer-crystal association but to polymer-ion association and subsequent mineral formation along these pre-organized templates, but this will have to be investigated in more detail in the future.

\section{Conclusion}

A set of electron microscopic techniques was used to elucidate structural and chemical details of biomimetic calcium phosphate nanofibers. The fibers are (as has been assumed earlier ${ }^{13,14,27-30}$ ) indeed crystalline calcium phosphate. Unlike previous assumptions, ${ }^{27,28}$ however, the calcium phosphate is not a single phase material. Rather it is a complex mixture of different calcium phosphates that are closely associated with the block copolymer template and the finer details of the crystal structure and possible sample preparation artifacts will need to be discussed in the future. In spite of this, the data indicate that the original model for calcium phosphate fiber formation $^{28}$ may need to be extended. Finally, the local $\mathrm{pH}$ within the polymeric template may differ by several orders of magnitude from the bulk value measured with the $\mathrm{pH}$ electrode, which is an aspect requiring quantification in the future. Overall, the data demonstrate that polycations are useful and complementary additives to the more commonly used polyanionic additives. They should not be disregarded when trying to understand biomimetic mineralization of calcium phosphate and other materials and may add value to the synthesis of advanced hybrid materials.

\section{Acknowledgements}

The authors thank Dr J. Reiche, Prof. G. Brezesinski, and Dr C. Stefaniu for access to the LB setups and for useful discussion, and Prof. H. Cölfen for providing Fig. 1C. We also thank an unknown, very critical reviewer for a number of very useful comments. M.J. acknowledges a doctoral fellowship from the MPI-KG. Funding from the University of Potsdam, the Max Planck Institute of Colloids and Interfaces (Colloid Chemistry Department), German Research Council, and the Karlsruhe Nano Micro Facility is gratefully acknowledged.

\section{References}

1 S. V. Dorozhkin, J. Mater. Sci., 2007, 42, 1061-1095.

2 S. V. Dorozhkin, Materials, 2009, 2, 399-498.

3 S. V. Dorozhkin, BIO, 2011, 1, 1.

4 S. V. Dorozhkin and M. Epple, Angew. Chem., Int. Ed., 2002, 41, 3130-3146.

5 M. Epple, Biomimetic bone substitution materials, 2007.

6 L. C. Chow, Dent. Mater. J., 2009, 28, 1-10.

7 C. Combes and C. Rey, Acta Biomater., 2010, 6, 3362-3378.

8 S. R. Paital and N. B. Dahotre, Mater. Sci. Eng., R, 2009, 66, $1-70$.

9 S. Schweizer and A. Taubert, Macromol. Biosci., 2007, 7, 1085-1099.

10 K. Bleek and A. Taubert, Acta Biomater., 2013, 9, 6283.

11 V. Ball, M. Michel, F. Boulmedais, J. Hemmerle, Y. Haikel, P. Schaaf and J. C. Voegel, Cryst. Growth Des., 2006, 6, 327-334.

12 A. Dey, P. H. H. Bomans, F. A. Müller, J. Will, P. M. Frederik, G. de With and N. A. J. M. Sommerdijk, Nat. Mater., 2010, 9, 1010-1014.

13 M. Junginger, K. Bleek, K. Kita-Tokarczyk, J. Reiche, A. Shkilnyy, F. Schacher, A. H. E. Müller and A. Taubert, Nanoscale, 2010, 2, 2440-2446.

14 M. Junginger, K. Kita-Tokarczyk, T. Schuster, J. Reiche, F. Schacher, A. H. E. Müller, H. Cölfen and A. Taubert, Macromol. Biosci., 2010, 10, 1084-1092.

15 P. A. Ngankam, P. Lavalle, J. C. Voegel, L. Szyk, G. Decher, P. Schaaf and F. J. G. Cuisinier, J. Am. Chem. Soc., 2000, 122, 8998-9004.

16 L.-J. Zhang, H.-G. Liu, X.-S. Feng, R.-J. Zhang, L. Zhang, Y.D. Mu, J.-C. Hao, D.-J. Qian and Y.-F. Lou, Langmuir, 2004, 20, 2243-2249.

17 O. Casse, O. Colombani, K. Kita-Tokarczyk, A. H. E. Müller, W. Meier and A. Taubert, Faraday Discuss., 2008, 139, 179-197.

18 E. D. Spoerke and S. I. Stupp, Biomaterials, 2005, 26, 5120-5129.

19 R. Löbbicke, M. Chanana, H. Schlaad, C. Pilz-Allen, C. Günter, H. Möhwald and A. Taubert, Biomacromolecules, 2011, 12, 3753-3760.

20 A. Shkilnyy, J. Brandt, A. Mantion, O. Paris, H. Schlaad and A. Taubert, Chem. Mater., 2009, 21, 1572-1578.

21 A. Shkilnyy, A. Friedrich, B. Tiersch, S. Schöne, M. Fechner, J. Kötz, C. W. Schlapfer and A. Taubert, Langmuir, 2008, 24, 2102-2109. 
22 A. Shkilnyy, R. Graf, B. Hiebl, A. T. Neffe, A. Friedrich, J. Hartmann and A. Taubert, Macromol. Biosci., 2009, 9, 179-186.

23 B. Cantaert, Y. Y. Kim, H. Ludwig, F. Nudelman, N. A. J. M. Sommerdijk and F. C. Meldrum, Adv. Funct. Mater., 2012, 22, 907-915.

24 D. Tsiourvas, A. Tsetsekou and M.-I. Kammenou, J. Am. Ceram. Soc., 2011, 94, 2023.

25 Y. B. Li, W. Tjandra and K. C. Tam, Mater. Res. Bull., 2008, 43, 2318-2326.

26 J. Yao, W. Tjandra, Y. Z. Chen, K. C. Tam, J. Ma and B. Soh, J. Mater. Chem., 2003, 13, 3053-3057.

27 M. Antonietti, M. Breulmann, C. G. Göltner, H. Cölfen, K. K. W. Wong, D. Walsh and S. Mann, Chem.-Eur. J., 1998, 4, 2493-2500.

28 A. Peytcheva, H. Cölfen, H. Schnablegger and M. Antonietti, Colloid Polym. Sci., 2002, 280, 218-227.

29 W. Tjandra, P. Ravi, J. Yao and K. C. Tam, Nanotechnology, 2006, 17, 5988-5994.

30 W. Tjandra, J. Yao, P. Ravi, K. C. Tam and A. Alamsjah, Chem. Mater., 2005, 17, 4865-4872.
31 Calcium Phosphates in Biological and Industrial Systems, Kluwer Academic Publishers, Norwell-Dordrecht, 1998.

32 A. A. Sousa, M. Hohmann-Marriott, M. A. Aronova, G. Zhang and R. D. Leapman, J. Struct. Biol., 2008, 162, 14-28.

33 D. B. Williams and C. E. Carter, Transmission Electron Microscopy - A Textbook for Materials Science, Plenum Press, New York, London, 1996.

34 R. Prakash, C. Wall, A. Kumar Mishra, C. Kübel, M. Ghafari, H. Hahn and M. Fichtner, J. Power Sources, 2011, 196, 5936-5944.

35 L. F. Drummy, C. Wang, R. Schoenmakers, K. May, M. Jackson, O. H. Koerner, B. L. Farmer, B. Mauryama and R. A. Vaia, Macromolecules, 2008, 41, 2135-2143.

36 D. Gebauer, A. Völkel and H. Cölfen, Science, 2008, 322, 1819.

37 Y. N. Liang, J. Hu, M. Kam Chiu Tam and X. Hu, RSC Adv., 2012, 2, 9531.

38 A. Shkilnyy, S. Schöne, C. Rumplasch, A. Uhlmann, A. Hedderich and A. Taubert, Colloid Polym. Sci., 2011, 289, 881 . 